Revue d'histoire de l'Amérique française

REVUE D.HISTOIRE DE L'AMÉRIQUE FRANÇAISE

\title{
La Coutume de Paris et la transmission des terres
}

Le rang de la Beauce à Calixa-Lavallée de 1730 à 1975

\section{Pauline Desjardins}

Volume 34, numéro 3, décembre 1980

URI : https://id.erudit.org/iderudit/303876ar

DOI : https://doi.org/10.7202/303876ar

Aller au sommaire du numéro

Éditeur(s)

Institut d'histoire de l'Amérique française

ISSN

0035-2357 (imprimé)

1492-1383 (numérique)

Découvrir la revue

Citer cet article

Desjardins, P. (1980). La Coutume de Paris et la transmission des terres : le rang de la Beauce à Calixa-Lavallée de 1730 à 1975 . Revue d'histoire de l'Amérique française, 34(3), 331-339. https://doi.org/10.7202/303876ar d'utilisation que vous pouvez consulter en ligne.

https://apropos.erudit.org/fr/usagers/politique-dutilisation/ 


\title{
LA COUTUME DE PARIS ET LA TRANSMISSION DES TERRES
}

\author{
LE RANG DE LA BEAUCE À CALIXA-LAVALLÉE DE 1730 À 1975*
}

\author{
PAULINE DESJARDINS \\ Étudiante, Département \\ d'anthropologie \\ Université de Montréal
}

Le patrimoine familial, selon Miner (1939), se transmet traditionnellement de père en fils au Canada français. Gérin (1889) avait observé cette tendance chez les cultivateurs de St-Justin. Toutefois, en réétudiant St-Justin, Garrigue a constaté que la majorité des terres n'appartenaient plus en 1955 aux familles qui les possédaient en 1889; elles ne passaient donc pas nécessairement de père en fils. Pour Garrigue, ce n'est pas la transmission intégrale du patrimoine qui en règle l'héritage, mais le désir des parents de s'assurer une retraite tranquille et d'établir les plus jeunes, quitte même à vendre à un étranger.

Cela expliquerait que, contrairement à l'esprit de la Coutume de Paris qui prévoit la répartition égalitaire des biens roturiers entre tous les enfants, la plupart des études démontrent la passation du patrimoine familial à un enfant seulement, tantôt à l'aîné, tantôt au cadet, tantôt au plus intéressé, les autres n'obtenant généralement que quelques biens meubles.

Afin de connaître comment se sont effectivement transmises les terres, nous avons fait l'étude des chaînes de titres de 15 terres dans le village de Calixa-Lavallée (anciennement Ste-Théodosie), situées dans la seigneurie de Verchères. Nous nous sommes rendue compte que c'est par le jeu des donations, celui de l'exclusion des enfants dotés et celui du principe de renonciation que l'on con-

* Texte d'une communication présentée au Congrès annuel de l'IHAF, tenu à l'Université d'Ottawa, les 19-20 octobre 1979. Les recherches nécessaires à la rédaction de ce texte ont été faites dans le cadre d'un mémoire de maîtrise.

RHAF, vol. 34, no 3, décembre 1980 
tourne l'esprit égalitaire de la loi en se protégeant ainsi contre la désintégration des patrimoines familiaux.

Le présent texte décrit notre méthodologie, rappelle les principes de base de la Coutume, en dégage les objectifs recherchés et les effets souhaités et signale au passage les moyens permettant de contourner les règles générales.

\section{Méthodologie}

Le choix de Calixa-Lavallée a été arbitraire. Le cahier no 4 de la série du Patrimoine (Calixa-Lavallée, Répertoire d'architecture traditionnelle) publie en annexe trois chaînes de titres et démontre la richesse de cette source pour l'étude des modes de transmission du patrimoine. Nous avons donc décidé d'approfondir cette recherche en nous basant sur Calixa-Lavallée, étant donné que les sources nous étaient fournies.

Pour un bref historique, reportons-nous au Répertoire:

Située dans le comté de Verchères, district de Montréal, la paroisse de Sainte-Théodosie devient officiellement CalixaLavallée le 7 septembre 1974 lors de la parution d'un décret dans la gazette officielle du Québec. - Partie intégrante de Verchères à l'origine, le rang de la Beauce est inclus dans la paroisse de Sainte-Théodosie en 1878. L'ensemble du territoire de Calixa-Lavallée est d'ailleurs lui-même détaché de la paroisse St-François-Xavier de Verchères et proclamé autonome le 24 juillet 1878. - Calixa-Lavallée est formé d'une partie de la troisième concession ou Petite Côte et des rangs de la Beauce, du Second-Ruisseau et du Petit Coteau. ${ }^{1}$

Notre interrogation de départ était: "Comment sont transmises les terres et à qui le sont-elles?» Pour répondre à cette question, plusieurs choix méthodologiques s'offraient à nous; entre autres, la possibilité de suivre une ou plusieurs familles de façon à contrôler l'ensemble du patrimoine dans le cadre des traditions familiales. Une autre possibilité était de suivre quelques terres en un endroit précis depuis leur concession, de façon à transcender les familles et à obtenir également une vue d'ensemble diachronique. Nous avions ainsi la possibilité de vérifier les influences françaises et anglaises, l'importance du morcellement des terres et plus parti-

1 Calixa-Lavallée, Répertoire d'architecture traditionnelle, Les Cahiers du Patrimoine, no 4, Direction générale du Patrimoine (MAC, Québec, 1977): 11. 
culièrement le jeu des solidarités familiales, tout en répondant à la question fondamentale.

Par cette dernière orientation, les chaînes de titres nous fournissent le matériel de base qui nous permet de connaître le «comment» (donation, cession, vente, testament, succession...) et le «à qui») (enfant ou étranger, garçon ou fille, aîné ou cadet ou autres). Ces chaînes de titres sont construites à partir des greffes de notaires et des documents conservés au Bureau d'Enregistrement. Les greffes des notaires de Verchères ont été systématiquement dépouillés pour la période d'avant 1850 , soit avant l'existence du Bureau d'Enregistrement. Pour la période subséquente, l'Index des immeubles ${ }^{2}$ nous fournit la chaîne de titres et les références aux actes notariés. L'Index des noms ${ }^{3}$ nous a également servi, mais avec certaines réserves, comme le mentionnent Séguin et Larouche. Chaque acte notarié se rapportant à une aliénation sur une des terres choisies a ensuite été dépouillé. Une reconstitution de chaque famille impliquée, actuellement en cours, nous fournira le complément d'information par rapport au lien de parenté et à l'ordre dans la famille (aîné, cadet, autre).

Nous avons choisi le rang de la Beauce à cause de l'ancienneté de son occupation, et plus particulièrement la partie où les lots sont bornés par le Ruisseau Codère afin de faciliter leur localisation. Ces lots, du moins pour la période d'avant l'installation du Bureau d'Enregistrement, sont juxtaposés, ce qui facilite l'identification des propriétaires avoisinants et évite ainsi les mauvaises pistes. Nous avons donc choisi quinze terres de chaque côté du Ruisseau Codère soit sept au nord-ouest, faisant partie du rang de la Beauce proprement dit, et huit leur faisant face du côté sud-est, identifiées au XVIIIe siècle comme continuation mais formant très vite le rang du Second Ruisseau. Il est certain que notre choix limite la généralisation; cependant, les premières concessions sur le rang de la Beauce datent de 1730 , ce qui assure une profondeur historique de 250 ans, soit de 10 à 12 générations, et de sept à huit cents transactions (594 transactions, soit environ $80 \%$ de l'échantillon total, ont servi à calculer les pourcentages préliminaires donnés dans ce texte). L'importance de l'échantillon est suffisante pour le rendre indicatif.

2 Normand Séguin et Daniel Larouche, «Les archives de l'enregistrement: commentaires sur les sources de la propriété foncière», Archives, 75,2: 131.

3 Ibid. 
Le présent texte fait part des premiers résultats de notre recherche en rapport avec la Coutume de Paris et de son influence sur la transmission. Dans son «Esquisse de la Coutume de Paris» ${ }^{4}$, Zoltvany nous en donne une très bonne description que nous ne reprendrons pas ici. Quant aux conclusions préliminaires et théoriques qu'il en tire, notre étude ne nous permet pas d'en contrôler les facteurs comme le douaire, le retrait lignager, la réserve des quatre-quints ou la légitime. Cependant, certains indices nous portent à croire que leur influence est minime. La cohésion de la cellule familiale ne nous apparaît pas aussi évidente que semble le croire Zoltvany. Les précautions prises par les parents lors des donations (description élaborée et précise des services que le donataire devra rendre aux donateurs, les obligations du donataire envers ses frères et soeurs) révèlent une grande méfiance. De plus, lors du rachat par un héritier de la part des autres, la passation d'un contrat notarié est aussi significative de cette méfiance, d'autant plus que la loi ne les y oblige pas. Quant à la deuxième conclusion de Zoltvany, l'importance des ventes, $60 \%$ de notre échantillon, montrent un fort dynamisme marchand. Nous reviendrons plus tard sur la signification de ce pourcentage. Mais déjà, on peut dire que, malgré le principe égalitaire qui sous-tend la Coutume, la transmission du patrimoine n'est pas égalitaire.

\section{Principes de base}

Parmi les principes de la Coutume qui influencent la transmission à Calixa-Lavallée, nous avons: le partage égalitaire entre tous les enfants, la protection des biens familiaux, la transmission en ligne directe. Ces principes s'exercent par le jeu des aliénations: à titre gratuit par donation ou testament, à titre onéreux par vente. Voyons maintenant comment joue chacun de ces types.

Le seul mode de transmission à cause de mort reconnu par la Coutume de Paris est la succession ab intestat qui répartit les biens du défunt, i.e. sa moitié de communauté, également entre tous les enfants. Les terres transmises sans testament ne représentent que $3 \%$ des transactions relatives aux terres étudiées. Cependant, la loi permet au père, administrateur des biens familiaux, d'aliéner ses biens par donation ou testament ou vente durant sa vie moyennant certaines contraintes. 384.

4 Yves Zoltvany, «Esquisse de la Coutume de Paris», RHAF, 25,3 (déc. 1971): 365- 
Les aliénations à titre onéreux, reliées au développement des transactions financières, vont à l'encontre de l'esprit coutumier de la loi, puisqu'elles permettent la circulation des biens à l'extérieur de la famille. La Coutume comporte donc des règlements très précis, tels l'hypothèque légale, le douaire de la femme et des enfants, les lods et ventes, le retrait lignager, afin de protéger les biens familiaux et d'éviter qu'ils ne soient dilapidés inopinément.

Cependant, certains mécanismes ont permis de minimiser ces règlements. La loi stipule que la présence de la femme à l'acte notarié représente une renonciation à l'hypothèque légale. Souvent même, lors de cet acte notarié, la femme renonce non seulement à l'hypothèque légale, mais également à son douaire et à celui de ses enfants sur le bien aliéné. Les lods et ventes dus au seigneur ne comptant que pour le douzième du prix de vente, leur importance n'est pas très restrictive; mais ils ont pu faire pencher la balance du côté des donations dans le cas des terres de grande valeur. Nous ne pouvons démontrer l'évidence de l'utilisation du retrait lignager. Quelques indices laissent entrevoir cependant que lors d'une faillite, par exemple, un membre de la famille essayait de récupérer sinon la totalité, du moins la majeure partie du patrimoine.

Les ventes représentent $60 \%$ des aliénations totales. Cependant, $90 \%$ de ces ventes ont eu lieu après 1880 , soit à l'époque de la formation du village, et se rapportent presque exclusivement à des aliénations sur les emplacements qui ne semblent pas constituer une partie importante du patrimoine familial et n'ont pas le caractère de continuité et de permanence des terres. De plus, une autre proportion de ces ventes est faite à l'intérieur de la famille: vente de droits de succession ou de terres entre frères et soeurs, vente de père à fils (au XXe siècle). Les ventes de droits de succession ou de parties de terre sont effectuées lorsque le père meurt avant de s'être démis de tous ses immeubles. Cette procédure permet d'éviter le morcellement tout en respectant la règle d'égalité. Les ventes entre père et fils apparaissent plus tardivement, soit au XXe siècle. L'adoption du Code civil en 1866, l'abolition du système seigneurial en 1854 et l'activité marchande sont sûrement les principales causes de ce nouveau type de transaction.

L'aliénation à titre gratuit, issue de la tradition mais non moins contrôlée, est restée pendant longtemps la principale forme d'aliénation. Elle était régie par le biais des modalités prévues pour 
les donations entre vifs et les testaments ${ }^{5}$. Cette deuxième catégorie d'aliénation doit respecter les principes de la Coutume. Ne donne pas qui veut et à qui il veut. Il est des personnes habilitées à recevoir et d'autres habilitées à donner. Les enfants naturels ne peuvent recevoir autre chose que des legs particuliers; les enfants adultérins et incestueux de même que les concubins ne peuvent recueillir autre chose que des aliments. Il est également défendu aux parents d'avantager leurs enfants dans des proportions inégales ou de s'avantager mutuellement s'ils ont des enfants.

Le testament ne peut être utilisé pour avantager un enfant au détriment des autres. Il est aussi limité par la légitime de droit et la réserve des quatre-quints sur les propres. Notre méthodologie ne nous permet pas de vérifier l'influence de ces restrictions, puisque nous ne contrôlons pas l'ensemble du patrimoine mais généralement seulement une partie, les habitants possédant d'autres terres dans la même seigneurie et dans les seigneuries avoisinantes. Sous le Régime anglais, les autorités, en rétablissant la Coutume de Paris, y introduisirent le mode testamentaire britannique basé sur la liberté testamentaire ${ }^{6}$. Toute personne saine d'esprit et majeure pouvait disposer, par testament, de tous ses biens meubles, immeubles, propres, acquêts ou conquêts - en faveur de qui que ce soit: conjoints, un ou plusieurs enfants, étrangers. La seule restriction s'appliquait aux corporations ou gens de main-morte non habilités à recevoir.

Les testaments observés, conformément à la Coutume de Paris, demeurent une forme d'aliénation limitée à des legs mineurs soit à l'Église, soit à des personnes désignées, et ce, jusqu'à la fin du XIXe siècle. On y décrit le type de sépulture, le nombre de messes à faire chanter, les legs mineurs en argent ou vêtements. Lorsque le legs testamentaire comprend des terres, on retrouve quelques testaments désignant le conjoint usufruitier et les enfants en propriété, ce qui respecte tout de même le principe de la Coutume de Paris cumulant les effets de l'hypothèque légale et du douaire. Ce n'est qu'après 1900 que le mode testamentaire anglais est vraiment utilisé à Calixa-Lavallée. On y désigne généralement le conjoint comme légataire universel, mais il y est souvent dénommé

5 L.-A. Jetté, «Des donations entre vifs et testamentaires», La Revue de droit, XV-XVI (1936-38).

6 André Morel, Les limites de la liberté testamentaire dans le droit civil de la Province de Québec (R. Pichon et R. Durand-Auzias, Paris, 1960), 176 p. 
comme précédemment en usufruit seulement. Les coutumes traditionnelles se perpétuèrent pendant très longtemps. On rencontre, dans les greffes consultés, des testaments en faveur de l'épouse ou de l'époux dès 1775 . On ne peut cependant dire si ces legs s'appliquent à des terres et s'ils sont égalitaires; une étude plus approfondie des testaments après la Conquête le précisera. Cette possibilité de tester en faveur de qui que ce soit permet l'inégalité: même si elle n'a pas été utilisée sous la forme de testament, elle a sûrement influencé l'inégalité dans les donations.

Les testaments ne prenant effet qu'à cause de mort, ils ne s'appliquent qu'aux biens restant à la mort du testateur. Il en est autrement des donations.

La donation entre vifs était utilisée depuis le Moyen Age surtout pour les dons à l'Église. L'utilisation courante de ce type d'aliénation qui n'était pas soumis à la réserve coutumière des quatre-quints était très dangereuse pour les héritiers naturels. La loi exigea que la donation fût faite pendant que le donateur était en santé, et qu'elle fit l'objet d'un acte notarié inscrit au Registre des Insinuations, de façon à réduire la possibilité d'influence ou de chantage sur une personne malade. Par la donation, un père pouvait avantager un enfant plus qu'un autre; cependant, à cause du principe d'égalité, la Coutume prévoyait que le donataire rapportait à la succession la valeur de la donation reçue. Le non-rapport équivalait à une renonciation à la succession.

Les enfants héritiers renoncent en effet à leurs legs et rapportent leur donation: l'égalité absolue règne entre eux. Mais comme ils peuvent renoncer à la succession, ils échappent à leur gré à la règle d'égalité.

La donation reste la forme préférée traditionnellement pour la transmission des terres, et la loi permettant l'inégalité des enfants, pour l'établissement de ces derniers. Souvent la donation stipule même que cette dernière est faite sans obligation de rapport et que le donataire pourra participer quand même à la succession. Cette exclusion des enfants dotés étudiée par Le Roy Ladurie ${ }^{8}$, était couramment employée en Normandie pour favoriser les fils,

7 Olivier Martin, Histoire de la Coutume de la Prévôté et Vicomté de Paris (2 vol., Paris, 1922-30, rééd. 1972), II: 425.

8 Emmanuel Le Roy Ladurie, «Système de la Coutume. Structures familiales et coutume d'héritage en France au XVIe siècle», Annales - Economies - Sociétés - Civilisations, 27, 4-5 (juillet-octobre 1972): 825-846. 
mais très peu dans la France de l'Ouest au XVIe siècle. Son utilisation à Calixa-Lavallée résulte-t-elle de la coutume normande ou d'un développement autochtone?

Les donations de terres à Calixa-Lavallée montrent une préférence pour les fils ou un fils en particulier. Les filles ont généralement des dots en biens meubles lors de leur mariage, i.e. mobilier de maison (buffet, lit garni, berceau), fournitures de maison (vaisselle, coutellerie, literie), animaux (moutons, vaches...), et argent. Les garçons auront des terres, des instruments aratoires, des bâtiments, des animaux et parfois aussi de l'argent. La donation aux filles se fait généralement lors de leur mariage, cependant que pour les garçons elle peut avoir lieu à n'importe quel moment au cours de leur vie.

Les donations ne représentent que $16 \%$ du nombre d'aliénations. Cette représentation ne décrit pas la réalité puisque la tendance qui semble se manifester est que les donations étaient courântes aux XVIIIe et XIXe siècles, mais qu'avec le XXe siècle, on semble préférer vendre la terre aux enfants, ou la léguer par testament. L'abolition du système seigneurial en 1854 et l'activité économique ont sûrement influencé ce changement ${ }^{9}$.

Le jeu des donations, celui de l'exclusion des enfants dotés et celui du principe de renonciation, permettent à un père de privilégier un ou plusieurs de ses enfants même si la loi prévoit que tous les enfants doivent recevoir des parts égales de l'héritage de leurs parents.

\section{Conclusion}

La Coutume de Paris qui voulait préserver les patrimoines familiaux et favoriser les solidarités familiales, permet malgré tout des libéralités allant à l'encontre de cet esprit. Par exemple, en donnant à l'épouse le pouvoir de renoncer à l'hypothèque légale et à son douaire par sa participation à l'acte d'aliénation, la loi enlève à cette dernière la protection qu'elle lui accorde et donne d'autre part au mari plein droit sur son épouse. La renonciation à la succession, qui a pour but de permettre aux enfants de se prévaloir contre une mauvaise administration de leur père, permet plus souvent qu'autrement une inégalité chez les enfants dans la répartition

9 Richard C. Harris, The Seigneurial System in Early Canada (Les Presses de l'Université Laval, Québec, 1966), 247 p. 
du patrimoine familial. L'exclusion des enfants dotés joue le même rôle et a le même effet.

En favorisant la transmission égalitaire du patrimoine entre tous les enfants, la Coutume contribuait en principe à la désintégration des patrimoines familiaux et au morcellement des terres. Il faut ajouter que cette règle d'égalité ne s'appliquait que pour les biens roturiers, les successions nobles allant pour la demie ou les deux tiers, selon le cas, à l'aîné mâle. La Coutume favorisait ainsi la désintégration des patrimoines familiaux roturiers et les empêchait de s'agrandir. La montée du mercantilisme au XVIIe siècle a permis de changer cette règle: c'est pourquoi on ajoute à la loi des clauses permettant de contourner cette règle d'égalité afin de préserver les petits patrimoines et même de les agrandir. Le jeu des donations et des échanges permettait à un père de donner, par exemple, les terres (immeubles) à ses fils et les biens meubles aux filles. Cependant le père qui ne possédait qu'une terre et voulait éviter de la morceler, pouvait la léguer à un de ses enfants, à charge pour celui-ci de dédommager ses frères et soeurs. Le père pouvait carrément tout donner à seulement un de ses enfants.

Les ordonnances émises sous le Régime français pour empêcher le morcellement des terres ont sûrement contribué à généraliser la transmission du patrimoine à un enfant en particulier. Sur 15 terres étudiées, deux ont été divisées pour agrandir les terres voisines, mais ce fut à la suite de ventes et non de legs familiaux. Les autres terres sont encore aujourd'hui approximativement ce qu'elles étaient lors de leur concession en 1730. Il faut toutefois mentionner que les gens du rang de «La Beauce» possédaient généralement plusieurs terres de culture et à bois permettant l'établissement de plusieurs enfants. En 1737, selon l'aveu et dénombrement, la seigneurie de Verchères était encore très peu peuplée et il y avait beaucoup de place pour l'établissement des jeunes. La proximité de Montréal et l'industrialisation du XIXe siècle ont attiré bien des jeunes laissant suffisamment de place pour ceux qui désiraient demeurer sur la terre. Enfin, l'achat par un enfant des parts des autres a aussi permis d'éviter le morcellement de ces terres.

En somme, si le mode testamentaire anglais n'a eu prise que très tardivement, c'est qu'en plus de l'attachement des paysans à leurs traditions, le mode coutumier de transmission des biens laissait la porte ouverte à une adaptation aux conditions de vie en évolution et à la possibilité d'agir selon leur volonté. 